PROCEEDINGS OF THE

AMERICAN MATHEMATICAL SOCIETY

Volume 133, Number 1, Pages 83-89

S 0002-9939(04)07669-5

Article electronically published on August 10, 2004

\title{
ON NEARLY ABELIAN POLYNOMIAL SEMIGROUPS
}

\author{
XIANTAO WANG AND ZHIGANG HUANG
}

(Communicated by Linda Keen)

\begin{abstract}
Let $G$ be a polynomial semigroup containing an element with degree at least 2 with the semigroup operation being functional composition. We prove that $G$ is nearly abelian if and only if the smallest $G$-completely invariant closed subset of the Riemann sphere is not equal to the Riemann sphere. We also give a positive answer to Conjecture 7.1 in Hinkkanen and Martin's paper on the dynamics of semigroups of rational functions.
\end{abstract}

\section{INTRODUCTION}

In the series of their papers, Hinkkanen and Martin tried to extend the classical theory of the dynamics associated to the iteration of a rational function of a complex variable to the more general setting of semigroups of rational functions; see [4] 5], etc.

Let

$$
f_{j}: \hat{C} \rightarrow \hat{C}(j=1,2, \cdots)
$$

be rational functions. We denote by

$$
G=\left\langle f_{1}, f_{2}, \cdots, f_{n}, \cdots\right\rangle
$$

the semigroup generated by the family $\left\{f_{j}: j=1,2, \cdots\right\}$ with the semigroup operation being functional composition.

The Fatou set of the semigroup $G$ is defined by

$$
F(G)=\{z \in \hat{C}: G \text { is normal in some neighbourhood of } z\}
$$

and the Julia set of $G$ by $J(G)=\hat{C} \backslash F(G)$. We write $F(f)$ and $J(f)$ for $F(\langle f\rangle)$ and $J(\langle f\rangle)$. Then $F(f)$ and $J(f)$ are the Fatou set and Julia set respectively, in the classical iteration theory of Fatou and Julia. It is obvious that the dynamics of a semigroup is more complicated than that of iteration of a single function. Some properties in the classical case cannot be preserved for the case of semigroups. For example, $F(G)$ and $J(G)$ may not be completely invariant, and $J(G)$ may not be $\hat{C}$ when $J(G)$ has an interior point; see the examples in [4].

Received by the editors August 15, 2003.

2000 Mathematics Subject Classification. Primary 30D05.

Key words and phrases. Polynomial semigroup, nearly abelian, completely invariant, Green's function.

This research was partly supported by FNS of China (No. 10271043), Soft Project of Science and Technology of Hunan Province and the Foundation for Scholars back from Foreign Countries.

(C)2004 American Mathematical Society Reverts to public domain 28 years from publication 
In order to extend Sullivan's famous result, non-existence of wandering domain ([8]), to the rational semigroups, Hinkkanen and Martin introduced a new notion.

A rational semigroup $G$ is called nearly abelian if there exists a compact set $\Phi(G)$ of Möbius transformations such that

(1) for any pair $\{f, g\} \subset G$, there exists an element (denoted by $[f, g]$ here and in the following) in $\Phi(G)$ such that $f \circ g=[f, g] \circ g \circ f$;

(2) for any pair $\{f, g\} \subset G,[f, g](F(G))=F(G)$.

They proved that Sullivan's theorem remains valid in such semigroups. In [7], Stankewitz defines the Julia set $J(G)$ of $G$ in a different way as follows:

$$
K(G)=\bigcap\{S \subset \hat{C}: S \text { is closed, } G \text {-completely invariant and } \operatorname{card}(S) \geq 3\},
$$

where a set $M$ is called $G$-completely invariant if it satisfies the following:

(1) $f(M) \subset M$ for any $f \in G$;

(2) $f^{-1}(M)=\{z: f(z) \in M\} \subset M$ for any $f \in G$.

Then, obviously, $K(G)$ is completely invariant and we have

Proposition 1.1. If $G$ contains an element whose degree is at least 2 , then $K(G)=$ $J(G)$ if and only if $J(G)$ is $G$-completely invariant.

In [7], Stankewitz proved

Theorem S. Let both $f$ and $g$ be two polynomials with degree at least 2 . If $J(f) \neq$ $J(g)$, then $K(\langle f, g\rangle)=\hat{C}$.

We will discuss this further and prove

Lemma 1.1. Let $G$ be a polynomial semigroup containing an element with degree at least 2. Then $K(G)=\hat{C}$ if and only if there exist two elements $f, g \in G$ with degree at least 2 such that $J(f) \neq J(g)$.

By using this generalization of Theorem $\mathrm{S}$, we will prove the following result in Section 2.

Theorem 1.1. Let $G$ be a polynomial semigroup containing an element with degree at least 2. Then $G$ is nearly abelian if and only if $K(G) \neq \hat{C}$ if and only if $\infty \notin$ $K(G)$.

Corollary 1.1. Let $G$ be a polynomial semigroup containing an element with degree at least 2 and $J(G)$ completely invariant. Then $G$ has no wandering domains.

Remark 1.2. Theorem 1.1 generalizes 4, Corollary 4.1 and Theorem 4.4] and [8. Theorem 2.1]. Also, Corollary 1.1 follows from the fact that $\infty \notin J(G)$, Proposition 1.1, Lemma 1.1 and [4, Theorem 5.1].

As the main result of this paper, we will prove in Section 3,

Theorem 1.3. If $G$ is a nearly abelian polynomial semigroup containing an element with degree at least 2 , then there exist a neighborhood $D$ of $\infty$ and a conformal mapping $B$ on $D$ such that

$$
B \circ G \circ B^{-1} \subset \Gamma=\left\langle z \rightarrow a z^{n}:|a|=1, n=1,2, \cdots\right\rangle,
$$

where $B \circ G \circ B^{-1}=\left\{B \circ f \circ B^{-1}, f \in G\right\}$.

Remark 1.4. In 4, Conjecture 7.1], Hinkkanen and Martin raised the question of whether there is a neighborhood of $\infty$ on which $G$ is analytically conjugate into the nearly abelian semigroup $\Gamma=\left\langle z \rightarrow a z^{n}:|a|=1, n=1,2,3, \ldots\right\rangle$ if $G$ is a nearly abelian polynomial semigroup. Theorem 1.3 is a positive answer to this question. 


\section{The Proof of TheOREM 1.1}

In order to prove Theorem 1.1, we need the following lemmas.

Lemma 2.1. Let $G$ be a polynomial semigroup containing an element with degree at least 2. Suppose that for any $f \in G$ with $\operatorname{deg}(f) \geq 2, J(f)=J(G)$. Then $J(G)$ is completely invariant.

Proof. Let $h \in G$. We need to treat two cases:

(i) Assume $\operatorname{deg}(h) \geq 2$. Then we have

$$
h(J(G))=h(J(h))=J(h)=J(G)
$$

and

$$
h^{-1}(J(G))=h^{-1}(J(h))=J(h)=J(G) .
$$

(ii) Assume $\operatorname{deg}(h)=1$. Take an $f \in G$ with $\operatorname{deg}(f) \geq 2$. Then we have $\operatorname{deg}(h \circ f) \geq 2$ and $J(f)=J(h \circ f)=J(G)$. By (i), we obtain

$$
J(G)=h \circ f(J(G))=h(J(G))
$$

and

$$
J(G)=(f \circ h)^{-1}(J(G))=h^{-1} \circ f^{-1}(J(G))=h^{-1}(J(G)) .
$$

This implies that $h^{-1}(J(G))=J(G)=h(J(G))$.

Lemma 2.1 follows from (i) and (ii).

Lemma 2.2. Under the assumptions of Lemma 2.1, for any $f, g \in G$, there exists $\phi(z)=a z+b$ with $|a|=1$ such that $\phi(J(G))=J(G)$ and

$$
f \circ g=\phi \circ g \circ f \text {. }
$$

Proof. Assume that neither the degree of $f$ nor the degree of $g$ is equal to 1 . Then by $[4$, Theorem 4.2], there exists $\phi(z)=a z+b$ with $|a|=1$ such that $f \circ g=\phi \circ g \circ f$.

Assume that $\operatorname{deg}(f)=\operatorname{deg}(g)=1$. Let $f(z)=a z+b$ and $g(z)=c z+d$, $a, b, c, d \in \hat{C}, a \neq 0$ and $c \neq 0$. Then $f \circ g(z)=a c z+a d+b$ and $g \circ f(z)=a c z+b c+d$. It follows that $f \circ g=\phi \circ(g \circ f)$ where $\phi(z)=z-b c-d+a d+b$.

For the remaining case, without loss of generality, we can assume that $\operatorname{deg}(f)=1$ and $\operatorname{deg}(g) \geq 2$. Take an $h \in G$ with $\operatorname{deg}(h) \geq 2$. Then

$$
\begin{aligned}
g \circ f \circ h & =\phi_{1} \circ f \circ h \circ g \\
& =\phi_{1} \circ f \circ \phi_{2} \circ g \circ h \\
& =\phi_{1} \circ \phi_{3} \circ \phi_{2} \circ f \circ g \circ h .
\end{aligned}
$$

This implies that $g \circ f=\phi_{1} \circ \phi_{3} \circ \phi_{2} \circ f \circ g=\phi \circ f \circ g$, where $\phi=\phi_{1} \circ \phi_{2} \circ \phi_{3}$.

By the discussion stated as above, for any $f, g \in G$, there exists $\phi(z)=a z+b$ with $|a|=1$ such that $f \circ g=\phi \circ g \circ f$. Combining this and Lemma 2.1, we can easily deduce that $\phi(J(G))=J(G)$.

The proof of Theorem 1.1. First of all, we need to prove Lemma 1.1. Let $K(G)=$ $\hat{C}$. Suppose that $J(f)=J(g)$ for any $f, g \in G$ with $\operatorname{deg}(f) \geq 2$ and $\operatorname{deg}(g) \geq 2$. By the proof of [4, Theorem 4.1] and Lemma 2.1, we have $J(f)=J(G)$ for any $f \in G$ with $\operatorname{deg}(f) \geq 2$ and $J(G)$ is completely invariant. Then $K(G) \subseteq J(G)$. This implies that $J(G)=K(G)$ and furthermore that $\infty \in J(G)$; so $\infty \in J(f)=J(G)$. But $f$ is a polynomial, and we have $\infty \in F(f)=F(G)$. This is a contradiction. Hence there exist two elements $f, g \in G$ with degree at least 2 such that $J(f) \neq$ $J(g)$. Combining this and Theorem S, we can deduce Lemma 1.1. Lemma 1.1 
XIANTAO WANG AND ZHIGANG HUANG

also gives the positive answer that $K(G) \neq \hat{C}$ if and only if $\infty \notin K(G)$. Since $K(G) \neq \hat{C}$ implies that $J(f)=J(g)$ for any $f, g \in G$ with $\operatorname{deg}(f) \geq 2$ and $\operatorname{deg}(g) \geq 2$, then by Lemma 2.1, $J(G)$ is completely invariant; thus $J(G)=K(G)$. Since $\infty \in F(f)=F(G)$ for some $f \in G$ with $\operatorname{deg}(f) \geq 2$, then we have $\infty \notin K(G)$. If $\infty \neq K(G)$, then it is obvious that $K(G) \neq \hat{C}$.

Now we are in a position to prove Theorem 1.1. If $G$ is nearly abelian, then by [4. Theorem 4.1], $J(G)=J(g)$ for each $g \in G$ of degree at least 2. Suppose that $K(G)=\hat{C}$. Then by Lemma 1.1 , there exist two elements $f, g \in G$ with degree at least 2 such that $J(f) \neq J(g)$. This is a contradiction. Thus $K(G) \neq \hat{C}$.

Suppose that $K(G) \neq \hat{C}$. Then by Lemma $1.1, J(f)=J(g)$ for any $f, g \in G$ with $\operatorname{deg}(f) \geq 2$ and $\operatorname{deg}(g) \geq 2$, and furthermore, $J(f)=J(g)=J(G)$ by the proof of [4, Theorem 4.1]. Then Theorem 1.1 follows from Lemmas 2.1 and 2.2.

\section{The Proof of Theorem 1.3}

Lemma 3.1. Let $f=a_{f} z^{n}+\cdots+a_{0}$ be a polynomial with $n=\operatorname{deg}(f) \geq 2$, and let $F_{\infty}$ denote the unbounded component of the Fatou set of $f$. Then there exist $D=\{z:|z|>R\} \subset F_{\infty}$ and a function $B(z)$, univalent in $D$,

$$
B(z)=z+b_{0}+\frac{b_{1}}{z}+\frac{b_{2}}{z}+\cdots \quad \text { in } \quad D,
$$

such that $|B(z)| \geq 1, f(D) \subset D$, and

$$
B \circ f \circ B^{-1}(z)=a_{f} z^{n}
$$

for all $z \in B(D)$. Then

$$
g_{F_{\infty}}(z, \infty)=\log |B(z)|+\frac{1}{n+1} \log \left|a_{f}\right|
$$

for all $z \in D$, where $g_{F_{\infty}}(z, \infty)$ denotes the Green function with respect to $F_{\infty}$ at $\infty$.

See 1] (p. 231), 2] (p. 105, p. 206) for its proof.

Without loss of generality, we can take an $f \in G$ with $\operatorname{deg}(f)=n \geq 2$ and $a_{f}=1$. Or we choose a suitable conjugate semigroup of $G$ such that this conjugate semigroup of $G$ contains an element $f$ with $\operatorname{deg}(f)=n \geq 2$ and $a_{f}=1$. By [6] or 1], there must exist $D=\{z:|z|>R\}$ for some $R>1$ and a univalent function $B(z)$ in $D$ such that

$$
B \circ f \circ B^{-1}(z)=z^{n} \text { and } \log |B(z)|=g_{F_{\infty}}(z, \infty) .
$$

Below, we will denote by $G$ a polynomial semigroup with a polynomial $f$ such that $\operatorname{deg}(f) \geq 2$ and $a_{f}=1$, by $B(z)$ the univalent function in Lemma 3.1 with respect to $f$, and by $D$ the neighborhood of $\infty$ in Lemma 3.1 with respect to $f$.

Lemma 3.2. If $G$ is nearly abelian, then for any $g \in G,\left|a_{g}\right|=1$.

Proof. For any $g \in G$, if $\operatorname{deg}(g)=k \geq 2$, then by [4, Theorem 4.1], $J(f)=J(g)$, i.e., $F_{\infty}=G_{\infty}$ where $F_{\infty}, G_{\infty}$ denote respectively the unbounded components of the Fatou sets of $f, g$. Then by Lemma 3.1, there exists a neighborhood $D^{\prime}$ of $\infty$ and a function $C(z)$ analogous to $B(z)$ that is univalent in $D^{\prime}$ such that

$$
g_{G_{\infty}}(z, \infty)=\log |C(z)|+\frac{1}{k+1} \log \left|a_{g}\right|,
$$


for all $z \in D^{\prime}$. Also, we can choose $D^{\prime}$ such that $D^{\prime} \subset D$. Since $F_{\infty}=G_{\infty}$, then $g_{F \infty}(z, \infty)=g_{G \infty}(z, \infty)$. By the uniqueness of the Green function,

$$
\log |B(z)|=\log |C(z)|+\frac{1}{k-1} \log \left|a_{g}\right|, \text { where } z \in D^{\prime} .
$$

From the expansions of $B(z)$ and $C(z)$, we have

$$
\frac{1}{k-1} \log \left|a_{g}\right|=0 \text {. }
$$

Hence $\left|a_{g}\right|=1$.

If $\operatorname{deg}(g)=1$, then $\operatorname{deg}(g \circ f)=\operatorname{deg}(f)=n \geq 2$. This implies that $\left|a_{g \circ f}\right|=$ $\left|a_{g}\right|=1$.

Lemma 3.3. Let $g \in G$ with $\operatorname{deg}(g)=k \geq 2$. If $G$ is nearly abelian and there exists $p>0$ such that

$$
B \circ g^{p} \circ B^{-1}(z)=a_{g^{p}} z^{k^{p}}
$$

for all $z \in B(D)$, then $B \circ g \circ B^{-1}(z)=a_{g} z^{k}$ for all $z \in B(D)$.

Proof. Since $\operatorname{deg}(g)=k \geq 2$, there exists a neighborhood $D^{\prime}$ of $\infty$ and a function $C(z)$ that is univalent in $D^{\prime}$ such that

$$
C g C^{-1}(z)=a_{g} z^{k}
$$

for all $z \in D^{\prime}$. Also, we can choose $D^{\prime}$ such that $D^{\prime} \subset D$.

Since $J_{g^{p}}=J_{g}$, that is $G_{\infty}^{p}=G_{\infty}$, where $G_{\infty}^{p}$ denotes the unbounded component of the Fatou set of $g^{p}$, by the uniqueness of Green's function over $G_{\infty}$, we know that

$$
\begin{aligned}
g_{G_{\infty}}(z, \infty) & =\log |C(z)|+\frac{1}{k-1} \log \left|a_{g}\right| \\
& =\log |B(z)|+\frac{1}{k^{p}-1} \log \left|a_{g^{p}}\right|
\end{aligned}
$$

for all $z \in D^{\prime}$. By Lemma 3.2, $\left|a_{g}\right|=1$ and $\left|a_{g^{p}}\right|=1$. This implies that $B(z) \equiv$ $C(z)$ in $D^{\prime}$. It follows from $B g B^{-1}(z)$ being analytic in $B(D)$ and $B g B^{-1}(z)=$ $C g C^{-1}(z)=a_{g} z^{k}$ in $B\left(D^{\prime}\right)$ that

$$
B g B^{-1}(z)=a_{g} z^{k}
$$

in $B(D)$.

Theorem 3.1. Let $G$ be a nearly abelian polynomial semigroup and $g \in G$ be an element with $\operatorname{deg}(g)=k \geq 2$. Then

$$
B g B^{-1}(z)=a_{g} z^{k}
$$

for all $z \in B(D)$.

Proof. Since $\infty$ is the superattracting point of $g$, and there exists $p>0$ such that $g^{p}(D) \subset D$, by Lemma 3.3 , we may assume that $p=1$.

By Lemma 3.1, we know that there exists $D^{\prime} \subset D$ and a function $K(z)$ analogous to $B(z)$ that is univalent in $D^{\prime}$ such that

$$
K \circ g \circ K^{-1}(z)=a_{g} z^{k}
$$

for all $z \in K\left(D^{\prime}\right)$. 
Since $J_{f}=J_{g}$, i.e., $F_{\infty}=G_{\infty}$, by the uniqueness of the Green function, we know that

$$
K(z) \equiv B(z)
$$

for all $z \in D^{\prime}$.

We know that $B \circ g \circ B^{-1}(z)$ is analytic in $B(D)$ and $B \circ g \circ B^{-1}(z)=K \circ g \circ$ $K^{-1}(z)=a_{g} z^{k}$ in $B\left(D^{\prime}\right)$. It follows that

$$
B \circ g \circ B^{-1}(z)=a_{g} z^{k}
$$

in $B(D)$.

Theorem 3.2. Assume that $G$ is nearly abelian. Let $h \in G$ be an element with $\operatorname{deg}(h)=1$. Then

$$
B \circ h \circ B^{-1}(z)=a_{h} z
$$

for all $z \in B(D)$.

Proof. Since $\operatorname{deg}(h \circ f)=\operatorname{deg}(f)=n \geq 2$, by Theorem 3.1, we know that

$$
B \circ(h \circ f) \circ B^{-1}(z)=a_{h} z^{n}
$$

for all $z \in B(D)$, i.e.,

$$
\begin{aligned}
\left(B \circ h \circ B^{-1}\right) \circ\left(B \circ f \circ B^{-1}(z)\right) & =B \circ h \circ B^{-1}\left(z^{n}\right) \\
& =a_{h} z^{n}
\end{aligned}
$$

for all $z \in B(D)$. Hence

$$
B \circ h \circ B^{-1}(z)=a_{h} z
$$

for all $z \in B(D)$.

The proof of Theorem 1.3. The proof of Theorem 1.3 follows from Theorems 3.1 and 3.2 .

\section{ACKNOWLEDGMENTS}

This research was started when the first author was an academic visitor in Tsinghua University. He thanks Professor Jianhua Zheng for his useful help and the Department of Mathematics for hospitality.

The authors also thank the referee for his (or her) careful reading of this paper and useful suggestions and Professor Linda Keen for her patience.

In particular, the first author thanks Professors Zhong Li and Shengjian Wu for their original inspiration to this paper.

\section{REFERENCES}

[1] I. N. Baker and A. Eremenko, A problem on Julia sets, Ann. Acad. Sci. Fenn. Series A. I. Math. 12 (1987), 229-236. MR0951972 (89g:30047)

[2] A. Beardon, Symmetries of Julia sets, Bull. London Math. Soc. 22 (1990), 576-582. MR:1099008 (92f:30033)

[3] A. Beardon, Iteration of rational functions, Springer, New York, 1988. MR 1128089 (92j:30026)

[4] A. Hinkkanen and J. Martin, The dynamics of semigroups of rational functions I, Proc. London Math. Soc. 73 (1996), 358-384. MF1397693 (97e:58198)

[5] A. Hinkkanen and J. Martin, Julia sets of rational semigroups, Math. Zeit. 222 (1996), 161169. MR 1429333 (98d:30038)

[6] G. Julia, Memoire sur la permutabilité des fractions rationelles, Ann. Sci. Ecole Norm. Sup. 39 (1922), 131-215. 
[7] R. Stankewitz, Completely invariant Julia sets of polynomial semigroups, Proc. Amer. Math. Soc. 127 (1999), 2889-2898. MR1600149 (2000a:37032)

[8] D. Sullivan, Quasiconformal homeomorphisms and dynamics I: Solution of the Fatou-Julia problem on wandering domains, Ann. of Math. 122 (1985), 401-418. MR0819553 (87i:58103)

Department of Mathematics, Hunan Normal University, Changsha, Hunan 410081, People's Republic of China

E-mail address: xtwang@mail.hunnu.edu.cn

Department of Mathematics, Tsinghua University, Beijing 100080, People's Repulic OF China

Current address: Department of Mathematics, University of Science and Technology of SuZhou, Suzhou, Jiangsu 215011, People's Republic of China

E-mail address: huang.z.g@263.sina.com 